\title{
The SV40 enhancer can be dissected into multiple segments, each with a different cell type specificity
}

\author{
Sabine Schirm, ${ }^{1}$ Josef Jiricny, ${ }^{2}$ and Walter Schaffner, ${ }^{1}$ \\ ${ }^{1}$ Institute für Molekularbiologie II der Universität Zürich, Hönggerberg, CH-8093 Zürich, Switzerland; ${ }^{2}$ Friedrich Miescher \\ Institut, PO Box 2543, CH-4002 Basel, Switzerland
}

The SV40 enhancer is known to be active in a wide variety of tissues and species. It contains a number of sequence motifs that can be bound by protein factors and whose integrity is essential for full enhancer activity. We have individually analyzed three synthetic oligonucleotides derived from sequences present within the SV40 enhancer: two oligonucleotides contain variants of the enhancer "core" sequence (designated corePVUII and coreC) and the third represents a region containing a decanucleotide homology to the immunoglobulin promoters/enhancers (designated SPHI). The oligonucleotides were multimerized and linked to a $\beta$-globin test gene. Transcripts of the test gene were analyzed following transient expression in 10 cell lines representing a broad spectrum of tissues. We show that each of the three short segments can individually act as an enhancer when present in multiple copies. None of these enhancers is ubiquitously active; however, each shows activity in a distinctive subpopulation of cell lines. This cell type specificity is most remarkable in the case of the two oligonucleotide segments containing the core sequences. One of these is primarily active in CV-1 cells, whereas the other exhibits a cell type specificity identical to that of the entire enhancer, possibly identifying it as the most important sequence element within the native SV40 enhancer. Our data suggest that a particular cell type specificity is typical for individual enhancer segments, and that enhancers of differing specificity can be assembled from the individual sequence motifs by combining them in different patterns.

[Key Words: SV40 enhancer; remote transcription control; cell type-specific transcription; synthetic oligonucleotides]

Received December 5, 1986; revised version received and accepted January 8, 1987.

The phenomenon of remote transcriptional control by cis-acting DNA elements was originally discovered in mammalian cells and described as the enhancer effect (Banerii et al. 1981; Moreau et al. 1981). The first enhancing DNA sequence identified was that from the simian virus 40 (SV40) genome. When linked to homologous and heterologous promoters, it was found to stimulate transcription in an orientation-independent manner, even from a position downstream of the gene (Banerii et al. 1981). Following this discovery, a great number of viral and cellular enhancers have been reported. However, the SV40 enhancer is the best characterized and the most often used example of a prototype enhancer. In contrast to enhancers which exhibit a strict cell type specificity (Banerii et al. 1983; Gillies et al. 1983; for review, see Gluzman 1985; Serfling et al. 1985) or, at least, a cell preference (de Villiers and Schaffner 1981; Laimins et al. 1982), the SV40 enhancer is known to act in a wide variety of tissues and hosts. In addition to its activity in mammalian cells, it also stimulates transcription when introduced into frog kidney ( $R$. Miller and W. Schaffner, unpubl.) or even algal cells (Neuhaus et al. 1984). The sequences important for SV40 enhancer activity have been investigated by studying deletion-duplication events (de Villiers et al. 1984; Swimmer and Shenk 1984; Weber et al. 1984; Herr and Gluzman 1985; Herr and Clarke 1986) and, in a more refined way, by site-directed mutagenesis (Zenke et al. 1986; see also Weiher et al. 1983). These studies supported our suggestion that enhancers generally represent a modular arrangement of short sequence motifs (Boshart et al. 1985; Dorsch-Häsler et al. 1985; Serfling et al. 1985), each interacting with a specific cellular transcription factor. The first indication for the relevance of cellular trans-acting factors to SV40 enhancer function came from in vivo competition studies (Schöler and Gruss 1984). Cellular protein factors physically interacting in vitro with the SV40 enhancer were subsequently detected by DNase I footprinting (Davidson et al. 1986; Wildeman et al. 1986). Additionally, Davidson 
et al. (1986) provided evidence for a cell type-specific binding of certain protein factors to overlapping sets of sequence motifs within the SV40 enhancer. Evidence for the binding of more than one factor to a given enhancer was also indirectly shown by Sen and Baltimore (1986), who found that different segments of the immunoglobulin kappa enhancer did not compete with each other in protein-binding studies.

Hints that a segment of an enhancer may have a cell type specificity different from the complete enhancer first came from studies of SV40 enhancer variants, as well as from analyses of enhancers within the polyomavirus and Herpesvirus saimiri genomes (de Villiers and Schaffner 1981; Schirm et al. 1985). An SV40 enhancer deletion variant that had duplicated a residual enhancer segment restored enough activity for growth in CV-1 kidney epithelial cells (Weber et al. 1984). However, when tested in a wider variety of primate cells, this duplicated enhancer variant was active only in CV-1 and in lymphoid cells (M. Pettersson and W. Schaffner, unpubl.). An altered cell type-specificity of a mutant SV40 enhancer was also quoted in Herr and Clarke (1986). In addition, a small segment of the enhancer of Herpesvirus saimiri has a pronounced cell type specificity distinct from that of the complete enhancer (S. Schirm and W. Schaffner, unpubl.). A weak but significant cell type preference had also been observed for each half of the polyomavirus enhancer when tested separately (Herbomel et al. 1984; see also Amati 1985).

For our analysis of small segments of the SV40 enhancer, we have exploited the observation that a high activity can result from multiple tandem copies of a subfunctional enhancer segment (de Villiers et al. 1984; Laimins et al. 1984; Weber et al. 1984) or even a short oligonucleotide (Veldman et al. 1985; our own data; B. Ondek and W. Herr, pers. comm.). We report that short sequences dissected from the SV40 enhancer each have a characteristic cell type specificity, which can be distinct from that of the complete enhancer. Furthermore, we show that these specificities can be different even between two segments containing motifs of high sequence homology. Our findings indicate that enhancers are composed of multiple sequence elements which, in principle, can substitute for each other, but in a cell typespecific manner. It also appears that nucleotides next to a characteristic enhancer motif can modulate the cell type specificity of the motif. In addition, the finding of cell type-specific activity of multimerized segments derived from an enhancer that as a whole is active in most cells suggests a complex interplay of enhancer sequence motifs and their cognate factors.

\section{Results}

Short oligonucleotides from the SV40 enhancer have enhancing activity by themselves

Three short segments of the SV40 enhancer that can interact with protein factors in the context of the complete enhancer (Wildeman et al. 1986) were dissected in order to analyze their enhancing activity individually and to investigate their cell type specificity (Fig. 1A). For the choice of these segments, we took into consideration the reports by Herr and colleagues $(1985,1986)$ and especially that of Zenke et al. (1986), which showed that the integrity of each of these regions is essential for full enhancer activity. Three segments, designated corePVUII, coreC, and SPHI, were synthesized as the doublestranded oligonucleotides shown in Figure 1B. The corePVUII-oligonucleotide was synthesized as a 22-bp dimer of an 11-bp sequence. The boundaries were chosen in such a way that its multimerization did not create a new junction but rather resulted in a circular redundancy of sequences naturally occurring in the SV40 enhancer. Thus, the 22-bp dimer of this DNA segment contains a continuous 14-bp sequence from the SV40 enhancer, and the stretch of alternating purines and pyrimidines (bp 258-265) is conserved (Fig. 1C, part a). Both the coreC- and the corePVUII-oligonucleotides include the "core" consensus sequence (Weiher et al. 1983). The monomer of the corePVUII-oligonucleotide contains no other sequences besides this homologous stretch (Fig. 1C, part b). In an initial experiment these oligonucleotides were ligated to generate multiple copies, and then inserted into the SmaI site of the vector pSVXB (Tognoni et al. 1985), upstream of the SV40 early promoter and $\mathrm{T}$-antigen gene. Plasmid was prepared from pools of bacterial clones (one pool for each of the three oligonucleotides) and transfected into different cell lines. Enhancer activity of the polymerized synthetic sequences was monitored by staining for $\mathrm{T}$-antigen with indirect immunofluorescence. In this assay the coreColigonucleotide was found to be active in all cell lines tested, namely CV-1 (monkey kidney), HeLa (human cervix carcinoma), Molt-4 (human T cells), and 3T6 (mouse fibroblasts), while the activity of corePVUII and SPHI were restricted to CV- 1 and lymphoid cells, respectively (data not shown). To follow up these preliminary observations in more detail, defined multimers (corePVUII, eight copies; coreC, four and eight copies; SPHI, eight copies) were inserted $1.2 \mathrm{~kb}$ downstream of a rabbit $\beta$-globin test gene in plasmid $\mathrm{JK}^{-}$(Fig. $\left.2 \mathrm{~A}\right)$.

A total of 10 different cell lines were transfected with these constructs, using either the calcium phosphate method (Graham and van der Eb 1973; Wigler et al. 1978) or the DEAE-dextran method (Luthman and Magnusson 1983), each with modifications according to the requirements of the cell line (see Materials and methods; for the origins of cell lines see Table 1). In all transfection experiments a negative control and a positive control were included (the expression vector containing no enhancer or the complete SV40 enhancer, respectively). A truncated rabbit $\beta$-globin gene under control of the SV40 enhancer was used as an internal control for transfection efficiency (Picard and Schaffner 1985). Enhancer activity was quantitated by S1 nuclease mapping of RNA from transfected cells. As a minimum, every cell line was transfected twice in independent experiments. The lymphoid cells each were tested three times, while the melanoma cell line Bowes and the rat 

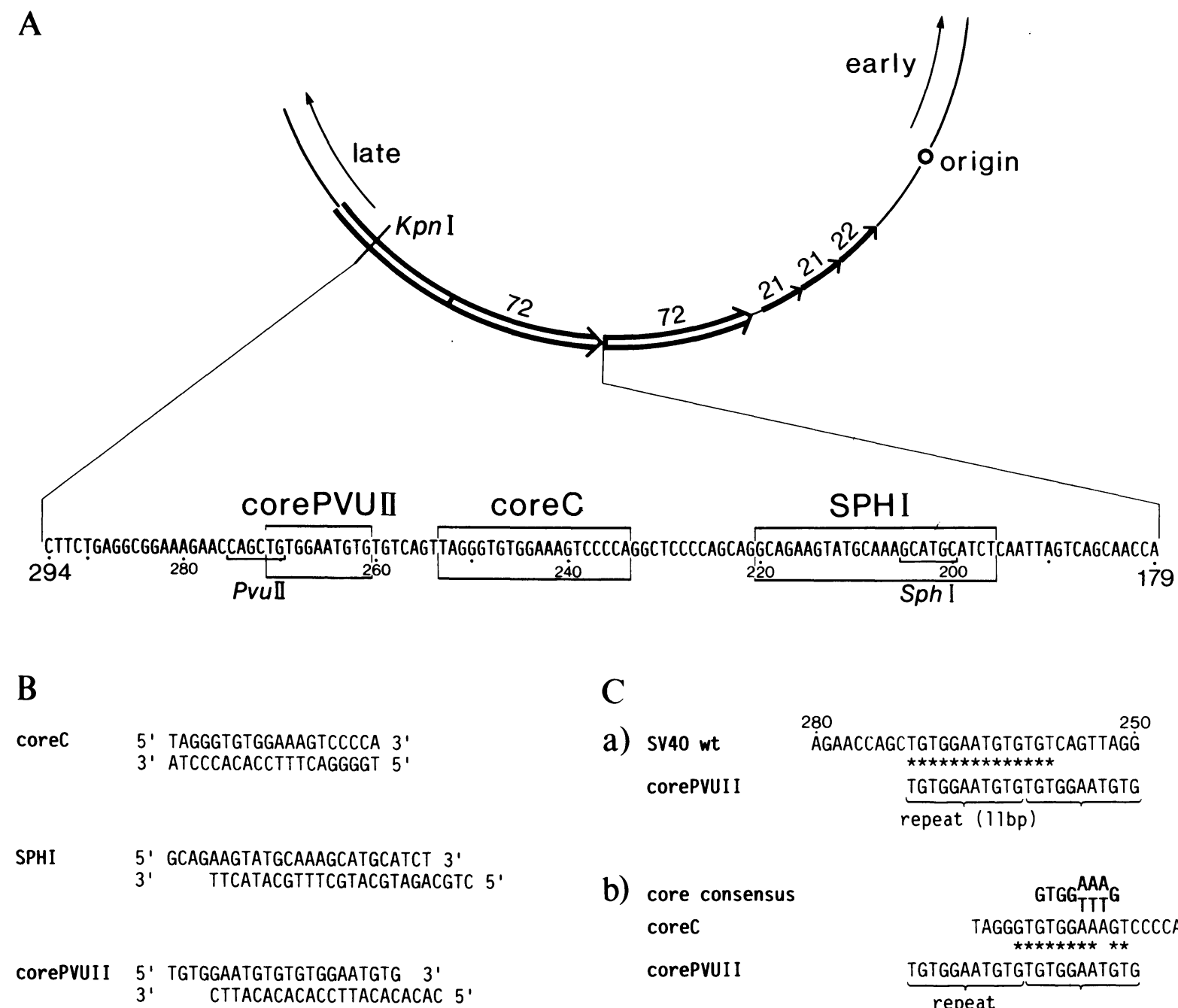

C

a) sV40 wt
corePVUII
b) core consensus
coreC
corePVUII
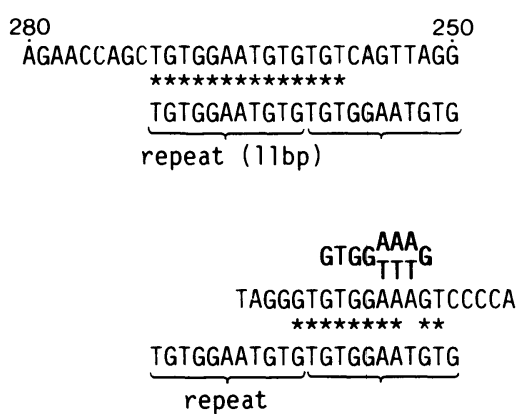

Figure 1. (A) Diagram of the SV40 regulatory region. The direction of late and early transcription, the origin of replication, and the $21 / 22$-bp promoter repeats are indicated. The enhancer is marked by a thick line; the nucleotide sequence of one 72 -bp repeat and flanking sequences extending to the KpnI site at nucleotide position 295 are shown (numbering according to Buchman 1981). Three synthetic oligonucleotides derived from the SV40 enhancer sequence were investigated (boxed). (B) Synthetic oligonucleotides. The sequences were synthesized as complementary double strands, either with blunt ends (coreC) or with a $5^{\prime}$ overhang of 4 bp (SPHI and corePVUII). $(C)$ Comparison of core consensus sequence motifs. (Part $a$ ) The corePVUII-oligonucleotide dimer is lined up underneath sequences of the SV40 enhancer. The dimer represents a continuous stretch of 14 bp from the wild-type enhancer (indicated by stars; for further explanation see text). (Part $b$ ) The homology between the coreC-and the core PVUII-oligonucleotides is indicated by stars. Note that the core consensus sequence (Weiher et al. 1983; on top) is fully contained in both oligonucleotides.

pituitary cell line GH3 B6 both were tested four times. In all cases the results were highly reproducible.

The core C-oligonucleotide is active in 9 out of 10 cell lines, as is the complete SV4O enhancer

In nine of the cell lines screened, the SV40 coreC-oligonucleotide $(20 \mathrm{bp}$; Fig. 1B) showed activity when present in four or eight copies. Interestingly, eight copies were considerably more than twice as active as four copies (Figs. 3 and 4 , lanes coreC4x and coreC $8 x$ ). Because core $\mathrm{C} 8 \mathrm{x}$ was created by dimerizing $\operatorname{coreC} 4 \mathrm{x}$, there are an extra four nucleotides between the two blocks of four copies (see Materials and methods). Though this changes the spacing between copies 4 and 5, we consider it unlikely that these extra four nucleotides contributed significantly to the enhancer effect with coreC8x. The enhancer effect with eight copies of coreC was at least as high as that of the whole SV40 enhancer, irrespective of the cell type and with only one exception (Figs. 3 and 4, lanes coreC8x and SV40 enh; Table 1). Surprisingly, in CV-1 cells, the cell line commonly used for lytic growth of SV40, eight copies of coreC were only about one-sixth as active as the SV40 enhancer as determined by densitometric scanning of the autoradiographs from two independent experiments. 


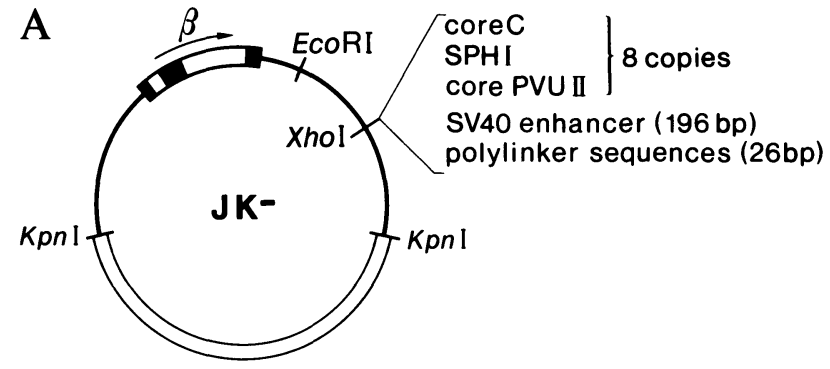

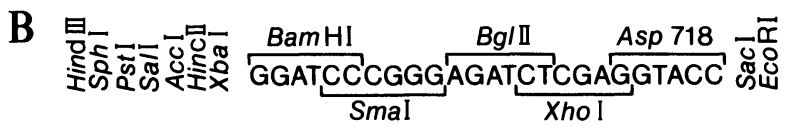

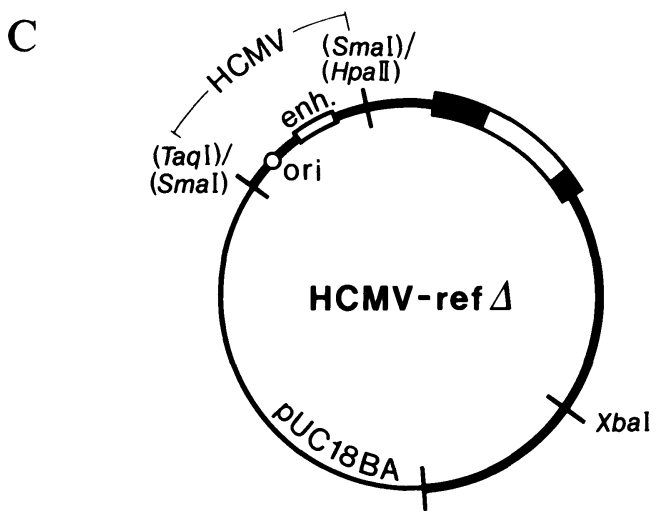

Figure 2. $(A)$ Structure of the recombinants analyzed in transient assays. Various DNA fragments were inserted into the $X$ hoI site of plasmid $\mathrm{JK}^{-}, 1.2 \mathrm{~kb}$ downstream of the rabbit $\beta$ globin gene. The activity of the coreC-, SPHI-, and corePVUIIoligonucleotides (each multimerized to eight copies) was compared with that of the SV40 enhancer (bp 100-295) and to a construct containing only polylinker sequences instead of an enhancer. $(B)$ Modified polylinker sequence of pUC18BA containing an additional restriction site for $B g l I I$ and $X h o I$ as compared with pUC18 (Yanisch-Perron et al. 1985). (C) Map of the internal reference gene clone containing the human cytomegalovirus enhancer.

Only a moderate enhancer effect was observed in Bowes melanoma cells (Fig. 3E); the SV40 enhancer and eight copies of the coreC-oligonucleotide stimulated transcription about 7 -fold and 15 -fold, respectively. In these cells, globin expression is quite high even in the absence of any enhancer, perhaps due to abundance of transcription factors interacting with the globin promoter. However, we cannot exclude the possibility that efficient transcription of the enhancerless $\beta$-globin gene is the result of intracellular recombination with the SV40 enhancer-containing reference plasmid.

Interestingly, and in contrast to all the other cell lines, the rat pituitary cell line GH3 B6 was negative for both the complete SV40 enhancer and the coreC-oligonucleotide (Fig. 5, lanes coreC4x, coreC8x and SV40 enh).
These findings confirm and extend those of A. Belayew and A.T. Truong (pers. comm.) and Camper et al. (1985), who have not seen any activity of the complete SV40 enhancer in these cells when assaying for expression of bacterial chloramphenicol acetyltransferase (CAT; Gorman et al. 1982). However, this effect is highly specific, and the coreC-oligonucleotide is not generally inactive in rodent cells, since the multimerized coreC-oligonucleotide is active in mouse $3 \mathrm{~T} 6$ cells when tested by immunofluorescence for $\mathrm{T}$ antigen (see above). For studies of enhancer activity in GH3 B6 cells, the Rous sarcoma virus enhancer (Weber and Schaffner 1985) and the human cytomegalovirus enhancer (Boshart et al. 1985) were used for a positive control and reference, respectively (Fig. 2C), as suggested by A. Belayew. With both of these enhancers, stimulation of transcription could be monitored easily in GH3 B6 cells (Fig. 5, lane RSV enh and data not shown), thus indicating that GH3 B6 cells are not, per se, incompetent to respond to a viral enhancer.

In all 10 cell lines of a broad spectrum of origin, there is a similar activity pattern of the SV40 enhancer and the coreC-oligonucleotide. Since other polymerized enhancer segments by themselves have a different cell type specificity (see below), it appears that the coreC-region is the dominant element of the SV40 enhancer. Especially the observation that both the complete enhancer and the coreC-oligonucleotide are inactive in the rat pituitary cell line GH3 B6 strongly supports this notion (for a summary of the results see Table 1).

Table 1. Activity of DNA segments dissected from the SV40 enhancer

\begin{tabular}{llclc}
\hline $\begin{array}{l}\text { Cell } \\
\text { Lines }\end{array}$ & Origin & SPHI & coreC & corePVUII \\
\hline CV-1 & monkey kidney & $1+1$ & 0.17 & 0.17 \\
HeLa & $\begin{array}{l}\text { human cervix } \\
\text { carcinoma } \\
\text { MCF7 }\end{array}$ & - & 2.8 & - \\
& $\begin{array}{l}\text { human breast } \\
\text { carcinoma }\end{array}$ & - & 2.1 & - \\
LN-18 & human glioma & $1+1$ & 1.1 & $1+1$ \\
Bowes & human melanoma & - & 2.5 & - \\
BJA-B & human B-cell & 0.32 & 2.1 & - \\
Namalwa & human B-cell & 1.4 & 1.2 & - \\
Molt-4 & human T-cell & 0.64 & 1.5 & - \\
D1112 & hybridoma & - & 1.4 & - \\
& $\quad$ human B-cell/ & & & \\
GH3 B6 & spleen) & & & - \\
\hline
\end{tabular}

Relative enhancer activity was determined quantitatively by densitometric scanning of the autoradiograms. The stimulation of transcription by the complete enhancer, relative to the reference gene, is referred to as 1 . Numeric representations of the activation by eight copies of the SPHI-, coreC-, and corePVUIIoligonucleotides are given relative to that of the SV40 enhancer and represent the average from two independent experiments. In cases of marginal $(+)$ or no enhancer activity we relied on visual inspection of the autoradiograms. 


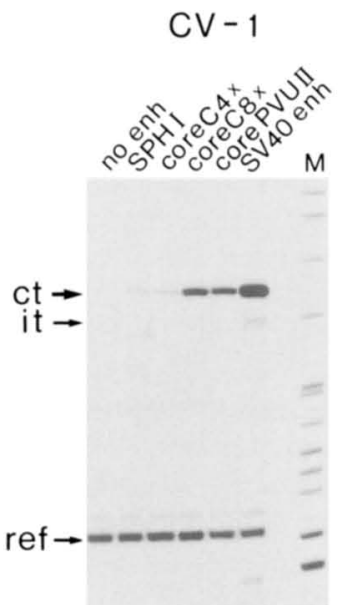

A

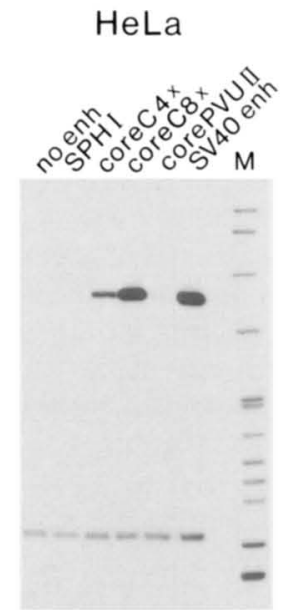

B

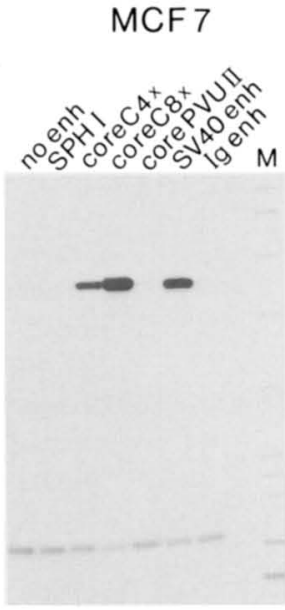

$\mathrm{C}$

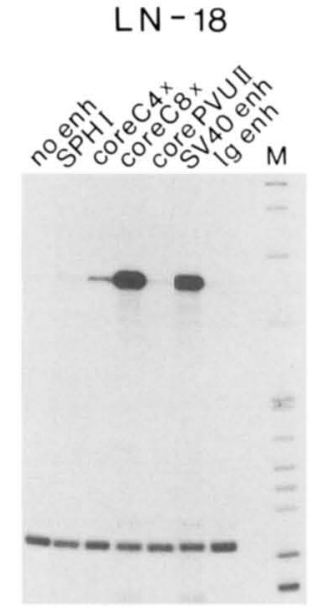

D
Bowes

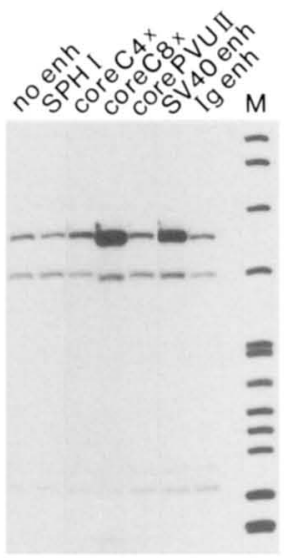

$\mathrm{E}$

Figure 3. Quantitative S1 mapping of rabbit $\beta$-globin RNA. The constructs containing multiple copies of oligonucleotides derived from the sequence of the SV40 enhancer (Fig. 2A) were transfected into different cell lines: CV-1 $(A) ; \mathrm{HeLa}(B) ; \mathrm{MCF} 7(C) ; \mathrm{LN}-18(D)$; Bowes $(E)$. In each panel the constructs investigated are arranged in the same order: (lane no enh) negative control plasmid without enhancer; (lane SPHI) eight copies of SPHI-oligonucleotide; (lane coreC4x) four copies of the coreC-oligonucleotide; (lane coreC8x) eight copies of the coreC-oligonucleotide; (lane corePVUII) eight copies of the corePVUII-oligonucleotide; (lane SV40 enh) complete SV40 enhancer; (lane Ig enh) 700-bp fragment (XbaI-EcoRI) of the mouse immunoglobulin heavy-chain enhancer (T. Gerster and W. Schaffner, unpubl.); (ct) correct terminus (354 nucleotides); (it) incorrect terminus (this band apparently results from initiation upstream of the rabbit $\beta$-globin cap site, followed by splicing into a cryptic 3' splice site at position +48 ; see de Villiers et al. 1982); (ref) truncated rabbit $\beta$-globin gene with SV40 enhancer (Picard and Schaffner 1985); $(M)$ marker DNA fragments (plasmid pBR322 cleaved with HpaII).

\section{Activity of the corePVUII-oligonucleotide is restricted} to $\mathrm{CV}$-1 cells

The corePVUII-oligonucleotide (Fig. 1B; synthesized as a 22-bp dimer of an 11-bp sequence) was further multimerized eight times to give a length similar to the eight copies of the other oligonucleotides. Due to sequence redundancy the dimer contains a 14-bp-long stretch of SV40 sequences (Fig. 1C, part a). It should be noted that the experiments described below were performed with a construct in which one of the dimers was inverted in orientation with respect to the others (the same cell type specificity was observed with a recent construct having all the monomers oriented head-to-tail; not shown). The multimerized corePVUII-oligonucleotide was highly active in CV-1 cells. However, no enhancer effect could be observed in any of the other cells lines, with the excep-

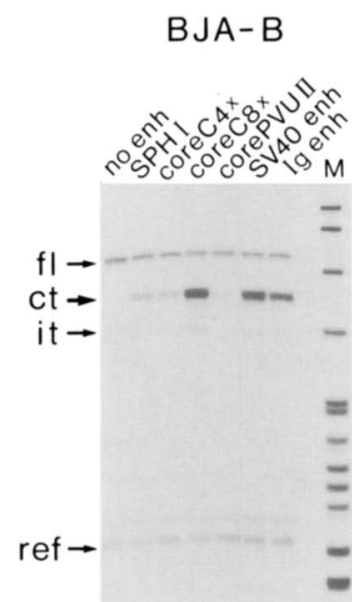

A

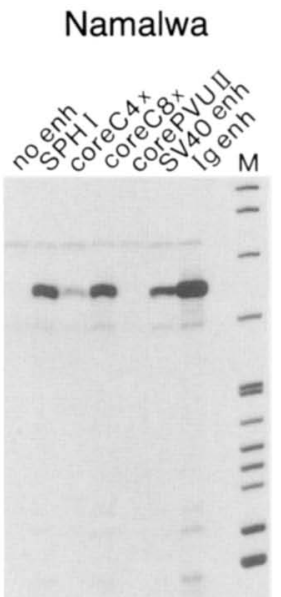

$\mathrm{B}_{1}$

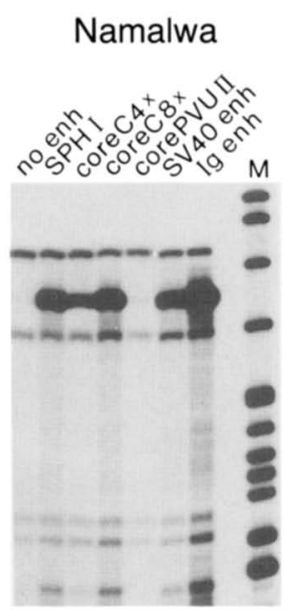

$\mathrm{B}_{2}$

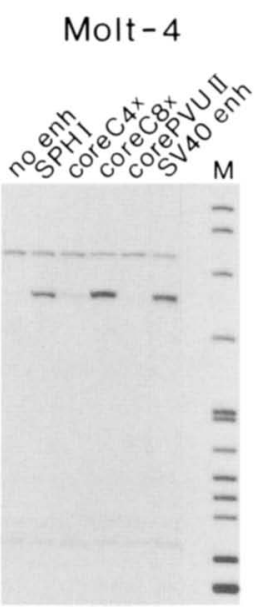

C
D1112

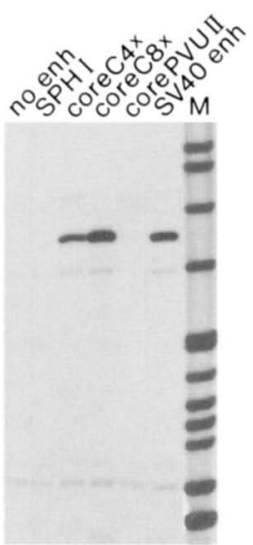

D

Figure 4. Quantitative S1 mapping of $\beta$-globin-specific RNA in lymphoid cells. The cell lines used in the study are: BJA-B $(A)$; Namalwa (B1 and B2) (the two panels represent different exposures of the same gel to show the reference gene); Molt-4(C); D1112 (D). The labeling of the lanes is the same as the Fig. 3; (fl) full-length probe (453 nucleotides). 


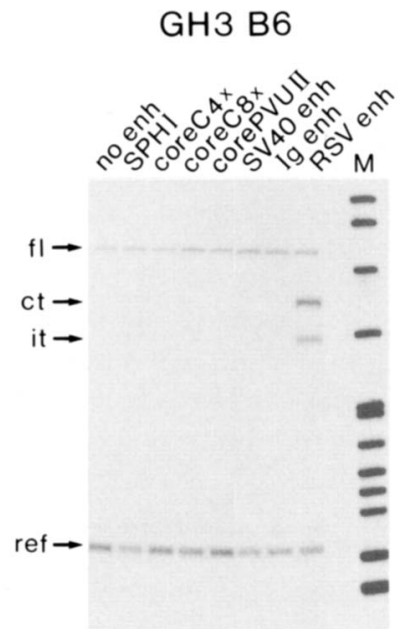

Figure 5. S1 mapping of $\beta$-globin-specific RNA in rat pituitary cells. The transfection of rat pituitary cells GH3 B6 was done with the same constructs as described in Fig. 3. (Lane RSV enh) Construct with the RSV enhancer downstream of the rabbit $\beta$ globin gene (Weber and Schaffner 1985); (fl) full-length probe (453 nucleotides); (ref) truncated $\beta$-globin gene with human cytomegalovirus enhancer.

tion of very low activity in LN-18 glioma cells (Figs. 3-5, lane corePVUII; Table 1). This was unexpected, since mutagenesis of this protein-binding sequence reduces activity of the complete SV40 enhancer when tested in HeLa cells (Zenke et al. 1986).

In CV-1 cells the corePVUII-oligonucleotide stimulates transcription to the same extent as the coreC-oligonucleotide (Fig. 3A, lanes coreC8x and corePVUII; Table 1). Thus, both oligonucleotides may contain sequence motifs which are equally important for the activity of the complete SV40 enhancer in this cell line. Interestingly, the cell type specificities of the coreC-and the corePVUII-oligonucleotides are very different. While coreC shows activity in a great variety of cells, the activity of corePVUII is restricted to CV-1 cells. Since both contain the core consensus sequence GTGGA/TA/TA/ TG /though with one base difference in the A/T segment; Fig. 1C, part b), it is likely that sequences next to the core consensus are responsible for the broad cell type specificity of the coreC element (see Discussion).

\section{SPHI-oligonucleotide shows lymphoid cell-specific activity}

The SPHI-oligonucleotide (25 bp) contains two direct repeats of $9 \mathrm{bp}$ of the sequence 5'AAGTATGCA/AAGCATGCA. These are essential for full activity of the complete SV40 enhancer in HeLa cells (Zenke et al. 1986) and bind factors from HeLa cell extracts (Wildeman et al. 1986). Interestingly, the junction of these direct repeats (ATGCA/AAGCA) is strongly homologous $(8 / 10 \mathrm{bp})$ to a motif that is conserved among immunoglobulin promoter and enhancer sequences /referred to as the decanucleotide; Falkner and Zachau 1984; Parslow et al. 1984). In another sequence context, this motif (also called octamer motif by some authors) is found in some housekeeping gene promoters (Harvey et al. 1982; Mattaj et al. 1985; Sive et al. 1986). No activity of the multimerized SPHI-oligonucleotide could be observed in HeLa cells (Fig. 3B, lane SPHI) despite the functional importance of this region within the native SV40 enhancer (Zenke et al, 1986). However, this DNA segment has an activity pattern identical to that of the lymphoid cell-specific immunoglobulin heavy chain $(\mathrm{IgH})$ enhancer (Banerii et al. 1983; Gillies et al. 1983; Neuberger et al. 1983): Both the multimerized SPHI-oligonucleotide of the SV40 enhancer and the mouse IgH enhancer were active in BJA-B, Namalwa, and Molt-4 cells (Fig. 4A, B, and C, lanes SPHI and Ig enh, and data not shown), whereas in D1112 cells, a cell line of lymphoid origin nonpermissive for the IgH enhancer, the SPHIsegment was inactive (Fig. 4D, lanes SPHI and not shown).

A short segment of the $\mathrm{IgH}$ enhancer containing the decanucleotide sequence parallels the tissue-specific activity of the complete IgH enhancer (T. Gerster and W. Schaffner, unpubl.; data not shown). This fragment therefore behaves similarly to the SPHI-oligonucleotide in this regard. Interestingly, in a band-shift assay (Strauss and Varshavsky 1984), both the IgH enhancer fragment and SPHI-oligonucleotide yielded identical tissue-specific patterns with extracts from $\mathrm{HeLa}$ and lymphoid (BJA-B) cell lines. Furthermore, the SPHI-oligonucleotide competed with the $\mathrm{IgH}$ enhancer fragment for the same protein factor in BJA-B and HeLa cells (P. Matthias and W. Schaffner, unpubl.; see also Discussion).

In addition to the activity in lymphoid cells, a low stimulation of transcription by eight copies of the SPHIsegment was found in the nonlymphoid cell lines CV-1 and $\mathrm{LN}-18$ (Fig. 3A and D, lanes SPHI). It remains to be determined whether the decanucleotide motif /which is probably responsible for activity of the SPHI-oligonucleotide in lymphoid cells) or adjacent sequences, distinct from those required in cells of lymphoid origin, are responsible for this activity.

\section{Discussion}

\section{Subsegments of the SV40 enhancer can act independently}

Our results show that multimers of short segments of the SV40 enhancer contain all necessary sequence information for enhancer activity. Sequence motifs within these segments seem to be protein-binding sites $(\mathrm{Da}$ vidson et al. 1986; Wildeman et al. 1986) whose integrity is required for full activity of the SV40 enhancer (Herr and Gluzman 1985; Herr and Clarke 1986; Zenke et al. 1986). We found that a high copy number of the individual oligonucleotides is needed for significant activity (compare lanes coreC $4 x$ and coreC8x, Figs. 3 and 4). This finding illustrates that a variety of different sequence motifs, as they are present in natural enhancers, are not required for the enhancer effect per se. There remains, however, a strong requirement for multiple sequence 
motifs, even if they are of the same type, for optimal enhancer activity.

\section{Broad cell type specificity of the coreC- but not the corePVUII-oligonucleotide}

The corePVUII-oligonucleotide corresponds to a subset of nucleotides within the coreC-oligonucleotide with only two mismatches. Both DNA segments contain a perfect match to the enhancer core consensus sequence GTGGA/TA/TA/TG (Fig. 1C, part b), which has been shown to be important for SV40 enhancer activity (Weiher et al. 1983; Herr and Clarke 1986; Zenke et al. 1986). Therefore, it is quite unexpected that these two oligonucleotides display such radically different, though overlapping, cell type specificities.

Several hypotheses that are amenable to experimentation could explain this observation. For example, the 20-bp-long coreC-oligonucleotide but not the shorter corePVUII-repeat might contain another protein-binding site in addition to, or overlapping with, the core factorbinding site, a situation which could of course influence cell type specificity. Alternatively, the core-binding protein could occur in multiple forms, either encoded by a family of related genes or as modified versions of one gene product. Different cell types would express a characteristic factor variant with a preferential affinity for a given core sequence. Furthermore, the affinity could conceivably be modulated by the nature of the nucleotides adjacent to the consensus sequence motif and/or the spacing between the motifs. Along this line of thought, the factor variant of most cells would efficiently bind to the coreC- but not the corePVUII-sequence. The factor characteristic for CV-1 cells, however, could bind to both sequences. At the same time this factor might have a relatively low affinity to the coreC-sequence, since we note that, only in CV-1 cells, eight copies of the coreC-oligonucleotide are less active than the SV40 enhancer (see Table 1).

\section{Preferential activity of the SPHI-oligonucleotide in lymphoid cells}

The SPHI-oligonucleotide contains a 9-bp direct repeat whose junction forms the decanucleotide motif which is also a typical component of immunoglobulin promoters and enhancers (Falkner and Zachau 1984; Parslow et al. 1984). Interestingly, the cell type-specificity of the multimerized SPHI-oligonucleotide correlates with that of a 47-bp segment of the IgH enhancer which is also active in lymphoid cells only. Both oligonucleotides contain the decanucleotide motif, but the IgH enhancer segment lacks the 9-bp repeat present in the SV40 enhancer. Within the IgH enhancer segment the decanucleotide motif plays a dominant role since its elimination results in both a severe downmutation and a loss of in vitro factor binding. In addition, the SPHI-oligonucleotide competes in vitro with the IgH segment for the decanucleotide-binding factor and both sequences show the same cell type-specific behavior in bandshift assays $(T$. Gerster, P. Matthias, and W. Schaffner, unpubl.). As the sequence corresponding to the decanucleotide is the only homologous DNA stretch between the SPHI-oligonucleotide and the short IgH enhancer segment, these results suggest that the decanucleotide sequence is not only responsible for the protein interaction seen in vitro but also for the in vivo activity of the SPHI-oligonucleotide in lymphoid cells.

In agreement with this, the integrity of the decanucleotide motif within the SV40 enhancer is of higher importance in MPC11 BU4 plasmacytoma cells than in HeLa cells, as was shown by others (Davidson et al. 1986; Zenke et al. 1986): mutagenesis of this motif from ATGCAAAGCA to ATTACAAGCA within the context of the complete enhancer reduced activity in MPC11 BU4 cells to $45 \%$ but only to $70 \%$ in HeLa cells. Conversely, integrity of the 9-bp direct repeat seems to be more important for SV40 enhancer activity in HeLa cells. In our construction, however, the 9-bp repeat apparently cannot contribute to enhancer activity in HeLa cells, which may be explained by a requirement for additional flanking sequences, or by incorrect spacing of the 9-bp repeat between tandem copies of the 25-bp-long SPHI-oligonucleotide.

\section{Interplay of sequence motifs in the context of the complete enhancer}

A simple model accounting for the differential activity of individual enhancer segments in various cell types assumes that each segment binds a different factor depending on the consensus motif contained within the segment and that the enhancer activity is then governed by the availability of the corresponding factor within a given cell type. Consequently, an enhancer specific for a particular cell type would have to contain a combination of sequence motifs for which binding factors are abundant in that cell. A generally active enhancer could result from a multitude of different sequence motifs, such that inactivity of any given motif is balanced by the activity of others.

We demonstrate that individual multimerized segments of the SV40 enhancer can act independently and with a strict cell type specificity, divergent from each other and from the complete enhancer. Using synthetic oligonucleotides provides the basis for gaining an understanding of the function of individual enhancer motifs and their interaction in the context of the natural enhancer. However, it also appears from our investigations that, in its natural context, a given sequence is important for full enhancer activity in a greater variety of cell types than after dissection. This seems to be the case for the corePVUII- and SPHI-oligonucleotides (compare our data to those of Davidson et al. 1986; Herr and Clarke 1986; Zenke et al. 1986). It is possible that these multimerized oligonucleotides are active by themselves only in certain cell lines that contain the cognate binding factors in high enough concentration or in a form of high affinity. In the majority of cell lines, however, binding of the corePVUII- and SPHI-specific factors might require some cooperativity with neighboring factors, a cooperativity that may be influenced by the spacing of sequence 
motifs (Hochschild and Ptashne 1986; see also Takahashi et al. 1986; see below). Further investigations, such as mutagenesis and footprint analysis, will be required to show the exact contribution of the sequence motifs to enhancer activity, and to exclude any influence of the junction sequence created by multimerization of the oligonucleotides. Also, there may be differently sized factor variants present in one or the other cell type. Thus, it will be interesting to elucidate whether there is an effect on activity and cell type specificity when varying the spacing between sequence motifs present in oligonucleotide repeats.

Contrary to what might have been expected, our experiments indicate that there is no obvious distinction between classes of "constitutive" and "cell type-specific" enhancers. Rather, a limited cell type specificity seems to be typical for individual enhancer segments. Comparing the similar coreC- and corePVUII-oligonucleotides, it appears that small variations in sequence and/or spacing of cis-acting regulatory elements can profoundly influence the cell type specificity.

Although we are only beginning to understand how enhancers of one or the other cell type specificity are assembled from combinations of different sequence motifs, we should ultimately be able to construct enhancers of desired cell type specificities by applying the principle of combination and its underlying rules. Also, as already shown for the enhancer containing eight copies of the coreC-oligonucleotide, such artificial enhancers can be considerably more active than their wildtype counterparts. Taken together, these findings should be of great value not only for basic research but also for therapeutic purposes involving cell type-specific effector delivery.

\section{Materials and methods}

\section{Cell culture}

Lymphoid cells D1112, BJA-B, and Namalwa (gifts from U. Weidle, Tutzing; H. zur Hausen, Heidelberg; C. Weissmann, Zürich, respectively/ were grown in RPMI 1640 medium supplemented with $10 \%$ fetal calf serum. For D1112 cells, the medium contained, in addition, $1 \mathrm{~mm}$ sodium pyruvate, $2 \mathrm{mM} \mathrm{L}$ glutamine and $1 \%$ nonessential amino acids. The other cell lines were propagated in Dulbecco's modified Eagles minimal medium (Gibco). For HeLa and CV-1 cells, the medium contained $2.5 \%$ calf serum and $2.5 \%$ fetal calf serum; for $3 \mathrm{~T} 6$, MCF7, LN-18, Molt-4, GH3 B6, and Bowes (gifts from W. Topp; G. Schütz, Heidelberg; N. de Tribolet, Lausanne; B. Fleckenstein, Erlangen; A. Belayew, Liege; J. Brüggen, Basel, respectively), the medium contained $10 \%$ fetal calf serum. Media were supplemented with $100 \mathrm{U} / \mathrm{ml}$ penicillin and $100 \mu \mathrm{g} / \mathrm{ml}$ streptomycin. The cells were seeded 1 day before transfection. At the time of transfection, $3 \mathrm{~T} 6$ cells were $20-30 \%$ confluent, the other adherent cells $50-70 \%$. Lymphoid cells /suspension cultures) had a density of $2 \times 10^{6}$ to $5 \times 10^{6}$ cells $/ \mathrm{ml}$ (in 100$\mathrm{mm}$ plates containing $10 \mathrm{ml}$ of medium).

\section{Construction of plasmids}

A tetramer of the coreC-oligonucleotide was cloned into the SmaI site of pSVXB (Tognoni et al. 1985) as a head-to-tail tandem. The EcoRI-XbaI fragment of this construct was then blunt-ended and cloned into the blunt-ended XhoI-cleaved expression vector $\mathrm{JK}^{-}$(Fig. 2A). The corresponding construct with eight copies of the coreC-oligonucleotide was obtained by dimerization of the insert of the above clone. The tetramer-containing plasmid was cleaved on either side of the insert with Asp-718 or BamHI, respectively, the protruding ends were removed with S1 nuclease, and the resulting blunt ends were fused. A spacer of $4 \mathrm{bp}$ was left between the two tetramer copies. The corePVUII- and SPHI-oligonucleotides were multimerized and blunt-ended, and the gel-extracted material was cloned as octamers into the SmaI site of pUC18BA (Fig. 2B; Yanisch-Perron et al. 1985); XhoI-Sall fragments from these constructs were then cloned into Xhol-cleaved $\mathrm{JK}^{-}$The orientation of the enhancer segment within the expression vector is the same for all constructs, as determined by Maxam-Gilbert sequencing (1980).

pUC18BA was constructed by replacing the BamHI-Asp 718 segment of the polylinker sequence in pUC18 (Yanisch-Perron et al. 1985) by a double-stranded oligonucleotide, thus introducing an additional restriction site for BglII and XhoI (Fig. 2B).

As a negative-control plasmid for the transfection experiments, polylinker sequences (XhoI-SalI segment of pUC18BA; Fig. 2B) were inserted into the $X$ hoI site of $\mathrm{JK}^{-}$.

For the construction of a reference gene with the human cytomegalovirus enhancer, a truncated rabbit $\beta$-globin gene with 425 bp of $5^{\prime}$-flanking sequences (Picard and Schaffner 1985) was introduced into pUC18BA. The enhancer-containing TaqIHpaII segment of the SV40-HCMV recombinant C4 (Boshart et al. 1985) was filled in by Klenow polymerase and inserted into the SmaI site of pUC18BA upstream of the globin gene (Fig. $2 \mathrm{C})$, resulting in a plasmid of about $7 \mathrm{~kb}$.

\section{DNA transfections}

Lymphoid cells were transfected by the DEAE-dextran protocol, as described by Luthman and Magnusson (1983), with modifications according to de Villiers and Schaffner (1983) and Banerii et al. (1983). After removal of the DNA/DEAE-dextran mixture, the cells were treated with $25 \%$ DMSO (Picard and Schaffner 1985). Rat pituitary cells GH3 B6 were incubated for $45 \mathrm{~min}$ at $37^{\circ} \mathrm{C}$ with $5 \mathrm{ml}$ of medium without serum (100-mm plates), containing $20 \mu \mathrm{g}$ DNA of the test gene, $5 \mu \mathrm{g}$ of the reference gene, and $200 \mu \mathrm{g} / \mathrm{ml}$ DEAE-dextran (F. Pasleau, pers. comm.). Transfections of the other adherent cell lines by the calcium phosphate coprecipitation followed the protocol by Graham and van der Eb (1973) and Wigler et al. (1978), with modifications described by Weber et al. (1984). For each transfection the test gene $(8.5 \mathrm{~kb})$ and the reference gene $(8.2 \mathrm{~kb})$ were used in the molar ratio $4: 1$ (except for the transfection of GH3 B6 where test and reference gene were used in the molar ratio $3: 1)$.

\section{RNA analysis}

Total cytoplasmic RNA was extracted $45 \mathrm{hr}$ after transfection (de Villiers and Schaffner 1983) and digested with proteinase K, and residual input plasmid DNA was removed by RNase-free DNase (Picard and Schaffner 1983). S1 nuclease mapping was performed with a single-stranded DNA probe labeled at the BamHI site (position 480) of a rabbit $\beta$-globin gene (Rusconi and Schaffner 1981) as described in Weaver and Weissmann (1979) and de Villiers and Schaffner (1983). Depending on the cell line, between 10 and $100 \mu \mathrm{g}$ of RNA were used for each analysis. (The level of transiently expressed $\beta$-globin-specific RNA was generally low for lymphoid cells and GH3 B6 cells.) 


\section{Acknowledgments}

We are especially grateful to Hans Rink (Ciba Geigy, Basel, Division of Biotechnology) for synthesizing the coreC-oligonucleotide and further oligonucleotides that were investigated in some initial experiments. We also thank Alexandra Belayew (Universite de Liege), Sart Tilman and Winship Herr (Cold Spring Harbor Laboratory, Cold Spring Harbor) for communication of results prior to publication, the colleagues mentioned in the text for gifts of cell lines, Werner Zürcher (Friedrich Miescher Institute, Basel) for help with oligonucleotide synthesis, Fritz Ochsenbein for the preparation of the figures, and Jutta Buxtorf for help in typing the manuscript. Finally, we acknowledge Dani Schümperli, Fred Schaufele, and Charles E. Samuel for critical reading of the manuscript. This work was supported by Schweizerischer Nationalfonds and the Kanton Zürich. S.S. was the recipient of an EMBO fellowship.

\section{Note added in proof}

After submission of our manuscript, we have learned that $\mathrm{W}$. Herr and his colleagues, in independent experiments, have observed different cell type specificities of individually polymerized segments of the SV40 enhancer (B. Ondek, A. Shepard, and W. Herr, EMBO J., submitted).

\section{References}

Amati, P. 1985. Polyoma regulatory region: A potential probe for mouse cell differentiation. Cell 43: 561-562.

Banerii, J., S. Rusconi, and W. Schaffner. 1981. Expression of a beta-globin gene is enhanced by remote SV40 DNA sequences. Cell 27: 299-308.

Banerii, J., L. Olson, and W. Schaffner. 1983. A lymphocyte-specific cellular enhancer is located downstream of the joining region in immunoglobulin heavy chain genes. Cell 33: 729740.

Boshart, M., F. Weber, G. Jahn, K. Dorsch-Häsler, B. Fleckenstein, and W. Schaffner. 1985. A very strong enhancer is located upstream of an immediate early gene of human cytomegalovirus. Cell 41: 521-530.

Buchman, A.R., L. Burnett, and P. Berg. 1981. The SV40 nucleotide sequence. In Molecular biology of tumor viruses, 2nd edition, revised: DNA tumor viruses. (ed. J. Tooze), pp. 799-841. Cold Spring Harbor Laboratory, Cold Spring Harbor, New York.

Camper, S.A., Y.A.S. Yao, and F.M. Rottman. 1985. Hormonal regulation of the bovine prolactin promoter in rat pituitary tumor cells. J. Biol. Chem. 260: 12246-12251.

Davidson, I., C. Fromental, P. Augereau, A. Wildeman, M. Zenke, and P. Chambon. 1986. Cell-type specific protein binding to the enhancer of simian virus 40 in nuclear extracts. Nature 323: 544-548.

de Villiers, J. and W. Schaffner. 1981. A small segment of polyoma virus DNA enhances the expression of a cloned beta-globin gene over a distance of 1400 base pairs. Nucleic Acids Res. 9: 6251-6264.

-1983. Transcriptional "enhancers" from papovaviruses as components of eukaryotic expression vectors. In Techniques in nucleic acid biochemistry (B5) (ed. R.A. Flavell), pp. B507/1-B507/20. Elsevier, Amsterdam.

de Villiers, J., L. Olson, C. Tyndall, and W. Schaffner. 1982. Transcriptional "enhancers" from SV40 and polyoma virus show a cell type preference. Nucleic Acids Res. 10: 79657976.

de Villiers, J., W. Schaffner, C. Tyndall, S. Lupton, and R.
Kamen. 1984. Polyoma virus DNA replication requires an enhancer. Nature 312: 242-246.

Dorsch-Häsler, K., G.M. Keil, F. Weber, M. Jasin, W. Schaffner, and U.H. Koszinowski. 1985. A long and complex enhancer activates transcription of the gene coding for the highly abundant immediate early mRNA in murine cytomegalovirus. Proc. Natl. Acad. Sci. 82: 8325-8329.

Falkner, F.G. and H.G. Zachau. 1984. Correct transcription of an immunoglobulin kappa gene requires an upstream fragment containing conserved sequence elements. Nature 310: $71-74$.

Gillies, S.D., S.L. Morrison, V.T. Oi, and S. Tonegawa. 1983. A tissue specific transcription enhancer element is located in the major intron of a rearranged immunoglobulin heavy chain gene. Cell 33: 717-728.

Gluzman, Y. 1985. Eukaryotic transcription. The role of cisand trans-acting elements in initiation. In Current Communications in Molecular Biology (ed. Y. Gluzman). Cold Spring Harbor Laboratory, Cold Spring Harbor, New York.

Gorman, C., L. Moffat, and B. Howard. 1982. Recombinant genomes which express chloramphenicol acetyltransferase in mammalian cells. Mol. Cell. Biol. 2: 1044-1051.

Graham, F.L. and A.J. van der Eb. 1973. A new technique for the assay of infectivity of human adenovirus 5 DNA. Virology 52: 456-467.

Harvey, R.P., A.J. Robins, and J.R.E. Wells. 1982. Independently evolving chicken histone $\mathrm{H} 2 \mathrm{~B}$ genes: Identification of a ubiquitous H2B-specific $5^{\prime}$ element. Nucleic Acids Res. 10: 7851-7863.

Herbomel, P., B. Bourachot, and M. Yaniv. 1984. Two distinct enhancers with different cell specificities coexist in the regulatory region of polyoma. Cell 39: 653-662.

Herr, W. and J. Clarke. 1986. The SV40 enhancer is composed of multiple functional elements that can compensate for one another. Cell 45: 461-470.

Herr, W. and Y. Gluzman. 1985. Duplications of a mutated simian virus 40 enhancer restore its activity. Nature 313: $711-714$.

Hochschild, A. and M. Ptashne. 1986. Cooperative binding of lambda repressors to sites separated by integral turns of the DNA helix. Cell 44: 681-687.

Laimins, L.A., G. Khoury, C. Gorman, B. Howard, and P. Gruss. 1982. Host-specific activation of transcription by tandem repeats from simian virus 40 and Moloney murine sarcoma virus. Proc. Natl. Acad. Sci. 79: 6453--6457.

Laimins, L.A., P. Tsichlis, and G. Khoury. 1984. Multiple enhancer domains in the $3^{\prime}$ terminus of the Prague strain of Rous sarcoma virus. Nucleic Acids Res. 12: 6427-6442.

Luthman, H. and G. Magnusson. 1983. High efficiency polyoma DNA tranfection of chloroquine treated cells. Nucleic Acids Res. 11: 1295-1308.

Mattaj, I.W., S. Lienhard, J. Jiricny, and E.M. De Robertis. 1985. An enhancer-like sequence within the Xenopus U2 gene promoter facilitates the formation of stable transcription complexes. Nature 316: 163-167.

Maxam, A.M. and W. Gilbert. 1980. Sequencing end-labelled DNA with base-specific chemical cleavages. Methods Enzymol. 65: 499-560.

Moreau, P., R. Hen, B. Wasylyk, R. Everett, M.P. Gaub, and P. Chambon. 1981. The SV40 72 base repair repeat has a striking effect on gene expression both in SV40 and other chimeric recombinants. Nucleic Acids Res. 9: 6047-6068.

Neuberger, M.S. 1983. Expression and regulation of immunoglobulin heavy chain gene transfected into lymphoid cells. EMBO I. 2: 1373-1378.

Neuhaus, G., G. Neuhaus-Url, P. Gruss, and H.G. Schweiger. 
1984. Enhancer-controlled expression of the simian virus 40 T-antigen in the green alga Acetabularia. EMBO J. 3: 21692172.

Parslow, T.G., D.L. Blair, W.J. Murphy, and D.K. Granner. 1984. Structure of the $5^{\prime}$ ends of immunoglobulin genes: A novel conserved sequence. Proc. Natl. Acad. Sci. 81: 2650-2654.

Picard, D. and W. Schaffner. 1983. Correct transcription of a cloned mouse immunoglobulin gene in vivo. Proc. Natl. Acad. Sci. 80: 417-421.

.1985. Cell-type preference of immunoglobulin kappa and lambda gene promoters. EMBO J. 4: 2831-2938.

Rusconi, S. and W. Schaffner. 1981. Transformation of frog embryos with a rabbit beta-globin gene. Proc. Natl. Acad. Sci. 78: 5051-5055.

Schirm, S., F. Weber, W. Schaffner, and B. Fleckenstein. 1985. A transcription enhancer in the Herpesvirus saimiri genome. EMBO I. 4: 2669-2674.

Schöler H.R. and P. Gruss, 1984. Specific interaction between enhancer-containing molecules and cellular components. Cell 36: 403-411.

Sen, R. and D. Baltimore. 1986. Multiple nuclear factors interact with the immunoglobulin enhancer sequences. Cell 46: $705-716$.

Serfling, E., M. Jasin, and W. Schaffner. 1985. Enhancers and eukaryotic gene transcription. Trends Genet. 1: 224-230.

Sive, H.L., N. Heintz, and R.G. Roeder. 1986. Multiple sequence elements are required for maximal in vitro transcription of a human histone H2B gene. Mol. Cell. Biol. 6: 3329-3340.

Strauss, F. and A. Varshavsky. 1984. A protein binds to a satellite DNA repeat at three specific sites that would be brought into mutual proximity by DNA folding in the nucleosome. Cell 37: 889-901.

Swimmer, C. and T. Shenk. 1984. A viable simian virus 40 variant that carries a newly generated sequence reiteration in place of the normal duplicated enhancer element. Proc. Natl. Acad. Sci. 81: 6652-6656.

Takahashi, K., M. Vigneron, H. Matthes, A. Wildeman, M. Zenke, and P. Chambon. 1986. Requirement of stereospecific alignments for initiation from the simian virus 40 early promoter. Nature 319: 121-126.

Tognoni, A., R. Cattaneo, E. Serfling, and W. Schaffner. 1985. A novel expression selection approach allows precise mapping of the hepatitis B virus enhancer. Nucleic Acids Res. 13: $7457-7472$.

Veldman, G.M., S. Lupton, and R. Kamen. 1985. Polyomavirus enhancer contains multiple redundant sequence elements that activate both DNA replication and gene expression. Mol. Cell. Biol. 5: 649-658.

Weaver, R.F. and C. Weissmann. 1979. Mapping of a RNA by a modification of the Berk-Sharp procedure: The $5^{\prime}$ termini of 15S beta globin mRNA precursor and mature $10 \mathrm{~S}$ beta globin mRNA have identical map coordinates. Nucleic Acids Res. 7: 1175-1193.

Weber, F. and W. Schaffner. 1985. Enhancer activity correlates with the oncogenic potential of avian retroviruses. EMBO $J$. 4: 949-956.

Weber, F., J. de Villiers, and W. Schaffner. 1984. An SV40 "enhancer trap" incorporates exogenous enhancers or generates enhancers from its own sequences. Cell 36: 983-992.

Weiher, H., M. König, and P. Gruss. 1983. Multiple point mutations affecting the simian virus 40 enhancer. Science 219: 626-631.

Wigler, M., A. Pellicer, S. Silverstein, and R. Axel. 1978. Biochemical transfer of single-copy eucaryotic genes using total cellular DNA as donor. Cell 14: 725-731.
Wildeman, A.G., M. Zenke, C. Schatz, M. Wintzerith, T. Grundström, H. Matthes, K. Takahashi, and P. Chambon. 1986. Specific protein binding to the simian virus 40 enhancer in vitro. Mol. Cell Biol. 6: 2098-2105.

Yanisch-Perron, C., J. Vieira, and J. Messing. 1985. Improved M13 phage cloning vectors and host strains: Nucleotide sequences of the M13mpl8 and pUC19 vectors. Gene 33: $103-119$.

Zenke, M., T. Grundström, H. Matthes, M. Wintzerith, C. Schatz, A. Wildeman, and P. Chambon. 1986. Multiple sequence motifs are involved in SV40 enhancer function. EMBO I. 5: 387-397. 


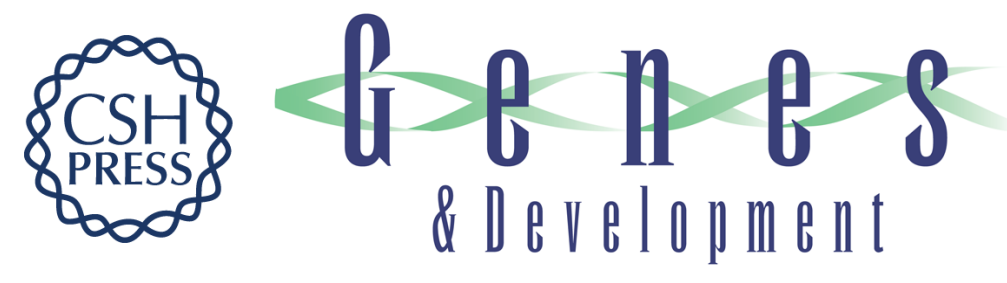

\section{The SV40 enhancer can be dissected into multiple segments, each with a different cell type specificity.}

S Schirm, J Jiricny and W Schaffner

Genes Dev. 1987, 1:

Access the most recent version at doi:10.1101/gad.1.1.65

References This article cites 49 articles, 12 of which can be accessed free at: http://genesdev.cshlp.org/content/1/1/65.full.html\#ref-list-1

License

Email Alerting Receive free email alerts when new articles cite this article - sign up in the box at the top Service right corner of the article or click here.

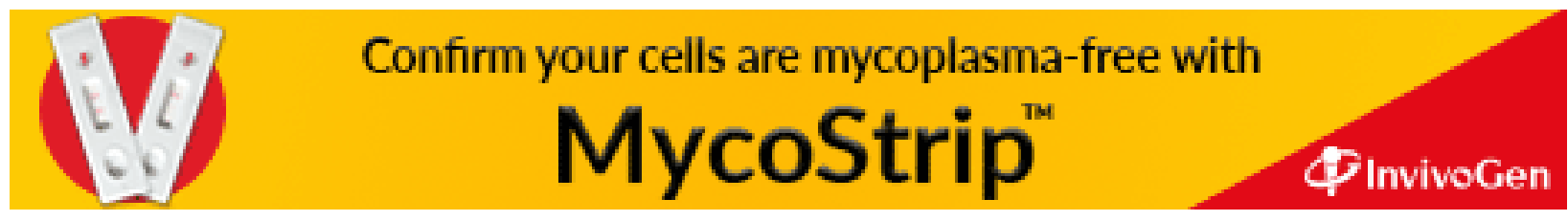

